\title{
Eingeblendete NS-Opfernarrative: Generationsübergreifende Latenz-Effekte in Literatur (Rymkiewicz, Wodin) und Comic (Hoven)
}

Opfernarrative stehen zunehmend unter Verdacht. Im besonderen Maße betrifft dies die von Norman G. Finkelstein mit seiner 2000 erschienenen Publikation sogenannte Holocaust Industry (2000 und 2001), die diesem Autor zufolge das jüdische Leiden - gelenkt von Verschwörungen in den USA - ausbeutet. Peter Novick, dessen The Holocaust in American Life (1999 und 2001) Finkelstein inspiriert hatte, führte die verkitschte Sakralisierung des Holocaust als einzigartiges Ereignis in die Opferdebatte ein. Beide Streitschriften wenden sich an ein amerikanisches Publikum, weshalb ihre deutschen Übersetzungen mit einem Vor- bzw. Nachwort der Autoren versehen sind, die auch deutlich machen, dass sich die Thesen nicht ohne Weiteres auf deutschsprachige Diskurse übertragen lassen. Zeitgleich machten Aleida Assmann und Ute Frevert in Geschichtsvergessenheit Geschichtsversessenheit: Vom Umgang mit deutschen Vergangenheiten nach 1945 (1999) auf die Inflation von Erinnerungsdebatten aufmerksam. Ihr voraus gingen im Nachkriegsdeutschland laut Assmann (2007) Etappen wie die anfängliche Erinnerungsabwehr zwecks Amnes(t)ierung der Täter`innen, die folgende Kritik an den blinden Flecken in der Vergangenheitsbewältigung und anschließende Erinnerungspolitik, die den Holocaust allgegenwärtig machte. Die hierbei ins Spiel gebrachten Vermarktungsstrategien liegen unter anderem in dem Dilemma begründet, dass gerade die Kulturindustrie die Erinnerung verbreitet, die hierdurch aber verstellt oder gar verzerrt wird (Werz 2014).

Angesichts der Fülle unterschiedlichster Narrationen, Erinnerungsorte und Gedächtnisveranstaltungen gerieten zwischenzeitlich die Opfer nicht nur unter Verdacht, sondern in Konkurrenz (Chaumont 2001; Franzen und Schulze Wessel 2012) um Anerkennung - selbst mit der Täterschaft - und mitunter in eine zukunftslähmende und kritikimmune Opferfalle (2015), wie der übersetzte Titel der Monografie des italienischen Literaturwissenschaftlers Daniele Giglioli lautet. ${ }^{1}$

Wie prekär der Opferstatus und -diskurs gerade im vom Nationalsozialismus bis heute traumatisierten Deutschland ist, verarbeitete die Regisseurin Frauke

1 Die italienische Originalausgabe erschien unter dem Titel Critica della vittima: Un esperimento con l'etica bei Nottetempo. 
Finsterwalder (2013) in ihrem Episodenfilm Finsterworld. Von den verwobenen Handlungssträngen seien exemplarisch die Verstrickungen genannt, die bei einer Klassenfahrt ins Konzentrationslager einen Kulminationspunkt finden, wenn zwei Schüler ihre Mitschülerin in einen Krematoriumsofen sperren, das von ihrem Lehrer befreite Mädchen hysterisch um sich schlägt, sodass ihre Bluse reißt, weshalb nun der Lehrer unter dem Verdacht versuchten sexuellen Missbrauchs steht. Befördert wird diese vermeintliche Täterschaft durch den eigentlichen Täter, einen Schüler, der, wie sich gegen Ende zeigt, unter der emotionalen Inkompetenz seiner wohlhabenden, posttraumatisierten Eltern leidet, die ihn materiell überversorgt, aber nie zärtlich umsorgt haben. Auch mehr als sechs Jahrzehnte nach Kriegsende, dies führt der mehrschichtige Generationenkonflikt in den filmischen Episoden und deren Schnittstellen vor, verkehren, überlagern und verzetteln sich Täterschaft und Opferstatus, Schuld und Leiden. Noch immer sind Traumata auszumachen, die Erinnerungen blockieren. Entsprechend kontrastieren die größtenteils in fast blendender Helligkeit gedrehten Szenen effektvoll mit den nicht-erzählten Dramen, mit dunklen Leerstellen in ungeklärten Familiengeschichten.

Im Nationalsozialismus erfolgt durch die Ablichtung von Opfern aus Tätersicht eine deutliche Rollenzuschreibung, indem das ,Schießen“ solcher Fotos an die drohende oder folgende Erschießung erinnert. Im Vergleich zum nationalsozialistischen Dokumentationswahn, der sich auch fotografisch niederschlägt, existieren sehr wenige Ablichtungen des Gräuels aus Opferperspektive. $\mathrm{Zu}$ den bekanntesten Beispielen zählen wohl die 1944 von Mitgliedern des Sonderkommandos heimlich angefertigten Aufnahmen aus Auschwitz-Birkenau. In Bilder trotz allem fasst Georges Didi-Huberman diese Fotos als Zeitzeugnisse auf, um sich auch teils spekulativ von Auschwitz „ein Bild [zu] machen“ (2007, 53). ${ }^{2}$ In der Studie, deren zweiter Teil eine ausführliche Auseinandersetzung mit der um 2000 vor allem aus Frankreich stammenden Kritik an dem fotografischen Spurenlesen darstellt, werden facettenreiche Bezüge, in denen Shoah-Fotografien entstanden und stehen, angeführt. Anhand der vier bis auf eine Ausnahme bereits seit 1945 bekannten und sehr unterschiedlich rezipierten Fotografien versucht Didi-Huberman „Fetzen“ $(2007,15)$ der Vergangenheit zu profilieren, die zwar keine allgemeingültige Wahrheit, aber jeweils eine vielschichtig spurenhafte Momentaufnahme sichtbar machten. Hierzu gehören etwa der Status und die Lesbarkeit der Fotografien als „,[produktiver] Akt des Widerstands in Auschwitz im Jahr 1944“ $(2007,93)$ oder die Auffassung vom „Bild als Riß, das einen

2 Zum fotografischen Bilderstreit zwischen diesem Autor und Claude Lanzmann im Kontext der Shoah-Erinnerung vgl. Gerstner 2013, 230-235. 
Schein des Realen auflodern läßt“ (2007, 121). Für Didi-Huberman sind es titelgebende Bilder trotz allem, weil „[d]iese Wendung [...] einen Riß zum Ausdruck [bringt]: Das alles verweist auf die Gewalt jener historischen Bedingungen, denen wir noch nichts entgegenhalten konnten; das trotz widersetzt sich dieser Gewalt durch die bloße heuristische Macht des Singulären“ (2007, 254). Eine Rezension zu Bilder trotz allem motivierte Gerhard Richter wiederum, die vier Fotografien als Vorlage seiner vierteiligen abstrakten Serie Birkenau von $2014 \mathrm{zu}$ wählen, worauf geschabte, gekratzte und verwischte Farbschichten die Ablichtungen bedecken. Einzig der Titel und die Werkgeschichte verdeutlichen Richters Opferdarstellung als Abstraktion bei seiner handwerklichen Übertragung der Fotos auf Leinwände. Bereits seit den 1960er Jahren setzt sich der Künstler mit Aufnahmen aus Konzentrationslagern auseinander, ohne jedoch zu einem ,handfesten' Ergebnis zu kommen. Richter wollte KZ-Fotografien in einer Ausstellung mit pornografischen Ablichtungen zeigen, um ganz im Sinne Susan Sontags auf die sich schnell einstellende voyeuristische Abstumpfung aufmerksam zu machen (Richter 2016; Sontag 1995, 26). Seit September 2017 hängen vier digitale Reproduktionen der Serie Birkenau im Westeingang des Bundestagsgebäudes. Nach Tal Sterngast

\footnotetext{
übermitteln [sie] eine Simulation der Gemälde, und stehen somit für einen weiteren Dreh im Hin und Her zwischen Fotografie und Malerei, dem Realen und dem Vorgestellten, der schon mit den Originalen der bearbeiteten Fotos aus Birkenau beginnt, deren Negative verschollen sind. Richter bringt die Fotos auf die Leinwand, und übersetzt sie in ein Foto zurück. Es ist typisch für Richters Arbeitsweise, eine metaphorische Glasscheibe zwischen Werk und Betrachter einzuziehen. (2017)
}

Hinzu kommt bei dieser Remedialisierung, dass sich die vier Bilder auf der Hochglanzoberfläche einer gegenüberliegenden vertikalen Flagge, d.h. von Richters Schwarz, Rot, Gold aus dem Jahre 1999 spiegeln. Für Nina Heindl und Véronique Sina fungiert Richters Birkenau-Serie als „Repräsentation ,trotz allem““ (2017), worin zugleich die Kluft zu den und die Schnittstelle mit den fotografischen Vorlagen besteht.

Ein ähnliches Verhältnis zu teils explizit erfundenen, teils in der Fiktion verbürgten Aufnahmen lässt sich in literarischen Opfernarrativen ausmachen, die aufgrund einer latenten Augenzeugenschaft von Nachkommen zwischen Sichtund Unsichtbarkeit, zwischen einer polysemantischen An- und Abwesenheit der Opfer oszillieren. Dies gilt insbesondere dann, wenn erzählt wird, was die Eltern- bzw. Großelterngeneration in den totalitären Systemen Anfang bis Mitte des zwanzigsten Jahrhunderts erlitt oder verbrach. In der nach Marianne Hirsch (1997, 2001, 2012) sogenannten ,Generation of Postmemory , der ersten, zweiten, dritten Nachgeneration, kommen mangels - oft selbst fotografischer - Augenzeugenschaft oder Erzählungen aus erster Hand, die allzu oft durch traumatische 
Amnesie und Aphasie bedingt sind, auffallend narrative Verfahren zum Einsatz, die ein breites mediales Spektrum aufweisen. Das großelterliche oder elterliche (Ver-)Schweigen erfährt hierbei eine aufschlussreiche mediale Kompensation, wodurch familiäre Erinnerungslücken ersetzt und hierbei explizit ausgestaltet werden. Aleida Assmann zufolge muss, was

zu einem bestimmten Zeitpunkt ausgeblendet, abgewiesen, ausgemustert oder verworfen ist, [...] noch nicht gänzlich verloren/vergessen sein: Es kann in materiellen Spuren gesammelt, aufbewahrt und einer späteren Epoche zugeführt werden, in der es neu gedeutet wird. $(2004,48)$

Mit Eintritt in das elektronische und digitale Zeitalter gewinnen „Echtheit und Authentizität“ (Assmann 2004, 57) von materiellen Wissensträgern eine neue Qualität. Auch in den postmemorialen Narrationen geraten manuell begreifbare ,Reliquien“ (Barthes 1970), die in direkter Berührung mit der Vergangenheit standen, in den Fokus. Letztgenannte verbürgen allerdings gerade keine Authentizität, sondern werden erzählerisch angesichts ihrer Zeugenschaft befragt, indem ihre Auslassungen akzentuiert werden.

Paradoxerweise manifestiert sich gerade in den Fehl- oder Leerstellen eine Latenz, die sich durch ihren Status des Potentiellen, durch Möglichkeiten des Daseins oder von Ereignissen auszeichnet. Im „Modus des Verborgenseins und der Wirksamkeit des Verborgenen“ (Diekmann und Khurana 2007, 9) lässt sich Latenz als solche nicht fassen, sondern einzig in ihren Effekten ausmachen, die aus Nachwirkungen von etwas Abwesendem resultieren. ${ }^{3}$ Da dieses Absente, das Unsichtbare in einer Vergangenheit begründet liegt, die als (narrativer) Referenzpunkt fungiert, schlägt sich das Latente gerade literarisch nieder (vgl. Gisbertz und Ostheimer 2017). Insbesondere transgenerationale Narrative können solche Latenzen ausfindig machen, um sie für Spekulationen über naheliegende, aber nicht verbürgte (Familien-)Geschichten zu nutzen. Die hiermit einhergehende Vergegenwärtigung spiegelt vor allem den Einfluss visueller Medien wider, die zugleich zeigen und ausschließen, wie wir dies von fotografischen Ausschnitten kennen, die immer auch durch das Nicht-Gezeigte bedingt sind. Zugleich komprimiert die Fotoaufnahme zeitlich eine Rück- und Vorschau gemäß dem Aorist, wie ihn Roland Barthes versteht: „Ich lese gleichzeitig: das wird sein und das ist gewesen“(1985, 106). Im Zeitalter omnipräsenter Vergegenwärtigung, die zunächst durch das Fernsehen, seit der Digitalisierung durch das Internet zur audiovisuel-

3 Hinsichtlich der zeitlichen Dimension konstatiert Thomas Khurana: „Das Latente ist keine zukünftige oder vergangene Gegenwart, es ist eine akut gegebene Ungegenwärtigkeit“ (2007, 143). 
len Alltagserfahrung wurde, wird selbst das Vergangene zunehmend aktualisiert. Auf welche Weise gerade Abwesendes, Leerstellen, Lücken und Auslassungen als latente Einblendungen in die Gegenwart von Schreibenden vordrängen, zeigen exemplarisch Auszüge aus Jarosław Marek Rymkiewiczs Umschlagplatz und Natascha Wodins Sie kam aus Mariupol, worin teils spekulative Opfernarrative durch eine fotografische Latenz angestoßen werden. Analog hierzu wird in Line Hovens Comic Liebe schaut weg gerade das Latente konturiert, um Aussparungen als solche auszuweisen und in ihrer Rätselhaftigkeit bestehen zu lassen, ohne jedoch die lückenhaft erzählte Familiengeschichte zu beklagen oder anzuklagen.

\section{Latente Opferbilder aus dem Off}

In einem aufschlussreichen Fokus stehen in Jarosław Marek Rymkiewiczs, Jahrgang 1935, ${ }^{4}$ Umschlagplatz von 1988 fotografische Posen aus dem Familienalbum. Angesichts des Titels ${ }^{5}$ weist der mit dem Autor gleichnamige und mit dessen autobiografischen Zügen ausgestattete Ich-Erzähler darauf hin, dass Historiker*innen sicherlich bessere Expert*innen für eine Rekonstruktion des in der Warschauer Topografie der 1980er Jahre verschwundenen Umschlagplatzes sind. Als Literaturhistoriker mit umfangreichen Interpretationserfahrungen sei er selbst jedoch auch prädestiniert, denn „Umschlagplatz nie istnieje i pozostały tylko opisujące go słowa“ (Rymkiewicz 1992, 41-42) [,,der Umschlagplatz existiert nicht mehr, geblieben sind nur mehr Worte, die ihn benennen“ (Rymkiewicz 1993, 60)]. In dem Text berichtet der Ich-Erzähler ebenso über seine Spurensuche nach dem Warschauer Umschlagplatz in literarischen Erinnerungen wie über seine kartografischen Rekonstruktionsversuche ${ }^{6}$ und topografischen Ortsbegehungen, um sich ein Bild zu machen. Umschlagplatz enthält zahlreiche fotografische Paralle-

\footnotetext{
4 Der Autor, etablierter Intellektueller, vor allem Dichter, ist zwischenzeitlich zur intellektuellen Galionsfigur der polnischen Rechtskonservativen avanciert. Vgl. hierzu die tendenziöse Filmdokumentation Poeta pozwany [Angeklagter Poet] (vgl. Braun 2012) sowie die im fundamentalistisch katholischen Fronda-Verlag erschienene Monografie Spór o Rymkiewicza [Der Streit um Rymkiewicz] (vgl. Rowiński 2012).

5 Bei dem Umschlagplatz in Warschau handelt es sich um eine Sammelstelle, wohin Jüdinnen aus dem Warschauer Getto zum Weitertransport in Vernichtungslager getrieben wurden. Zuvor war der Umschlagplatz ein Güterbahnhof. Der Ort des Grauens wurde 1988 zur offiziellen Gedenkstätte mit einem Monument. Literarisch setzte ihm 1946 bereits der polnische Pianist und Komponist Władysław Szpilman in seiner Autobiografie ein Denkmal (Szpilman 1946; Szpilman 2004), das auch Roman Polanski in seiner Verfilmung aufgriff (Pianista 2002).

6 Entsprechend ist in der polnischen Ausgabe ein Plan des Umschlagplatzes (Rymkiewicz 1992,
} 
len zu den Recherchen, von denen im vorliegenden Beitrag nur diejenigen aufgegriffen werden können, die die Schilderungen am Anfang und Ende des Buches flankieren.

$\mathrm{Zu}$ Beginn spekuliert der Ich-Erzähler über Kindheitsaufnahmen, die im Sommer 1942 an unterschiedlichen Erholungsorten in der Nähe Warschaus, in der idyllischen Natur und Peripherie der okkupierten Hauptstadt entstanden. Zu sehen sind hierauf die schaukelnde Schwester, das Geschwisterpaar am Flussstrand und der Bruder „na peronie w Otwocku. Krawat i czapeczka [i] [...] te białe skarpetki“ (Rymkiewicz 1992, 22) [,,auf dem Bahnsteig in Otwock. Mit Krawatte und Mütze [und] [...] diese weißen Socken“ (Rymkiewicz 1993, 31)]. Laut Datierung der Mutter stammt das Foto vom 19. Juli 1942, als es dem Ich-Erzähler zufolge dort noch ein Getto gab (Rymkiewicz 1992, 22; Rymkiewicz 1993, 31). ${ }^{7}$ Daher offenbart der Ich-Erzähler seiner Schwester, dass er ihre gemeinsame glückliche Kindheit angesichts der diametral entgegengesetzten Parallelwelt in wenigen Kilometern Entfernung als „coś nieprzyzwoitego“ (Rymkiewicz 1992, 23) [„ungehörig“ (Rymkiewicz 1993, 32)] empfindet und nimmt hierbei imaginativ die Perspektive der in ihren Ferien Abgelichteten ein, die unbeschwert in die Kamera blicken, während „w odległości nie większej niż cztery kilometry odbywa się to, co się odbywa“ (Rymkiewicz 1992, 23) [,,in einer Entfernung von nicht mehr als vier Kilometern geschieht, was damals geschah" (Rymkiewicz 1993, 32-33)]. Indem die fotografischen Posen des Geschwisterpaars, welche ungetrübte Kindheitstage bezeugen, immer wieder mit dem Gräuel in unmittelbarer Nähe, dem, „co tam się odbywało“ (Rymkiewicz 1992, 26) [„,was geschehen ist“ (Rymkiewicz 1993, 37)], kontrastiert werden, erlangen die Aufnahmen eine belastende Polyvalenz des Sichtbaren. Derart literarisiert gerät gerade das damalige Nicht-Sehen, das, was im fotografischen Off blieb, in den latenten Fokus. In Analogie hierzu stehen zum einen die Versuche, den topografisch verschwundenen, aber sprachlich dokumentierten Umschlagplatz zu rekonstruieren - ein Ort, der sich in ähnlicher Weise in ambiger Abwesenheit befindet wie die Gräueltaten auf den Kindheitsfotos. Zum anderen lösen gerade Letztgenannte imaginativ eine fotocollagenhafte Gegenüberstellung aus, welche den Ich-Erzähler von der idyllischen Peripherie an den Ort des damaligen Gräuels zurückführt: „Zdjęcie, które wszyscy znają: chłopiec w

8-9), in der deutschen Übersetzung zudem des Warschauer Gettos abgedruckt (Rymkiewicz 1993, 10-11).

7 Über das Getto von Otwock schreibt Calel (Calek) Perechodnik in seiner persönlichen Chronik von 1943, in der er sich selbst die Schuld an dem Tod seiner Frau und seiner kleinen Tochter zuweist, da er allzu leichtgläubig und naiv meinte, beide seien durch seine Tätigkeit als GettoPolizist geschützt (Perechodnik 1993, 2015). 


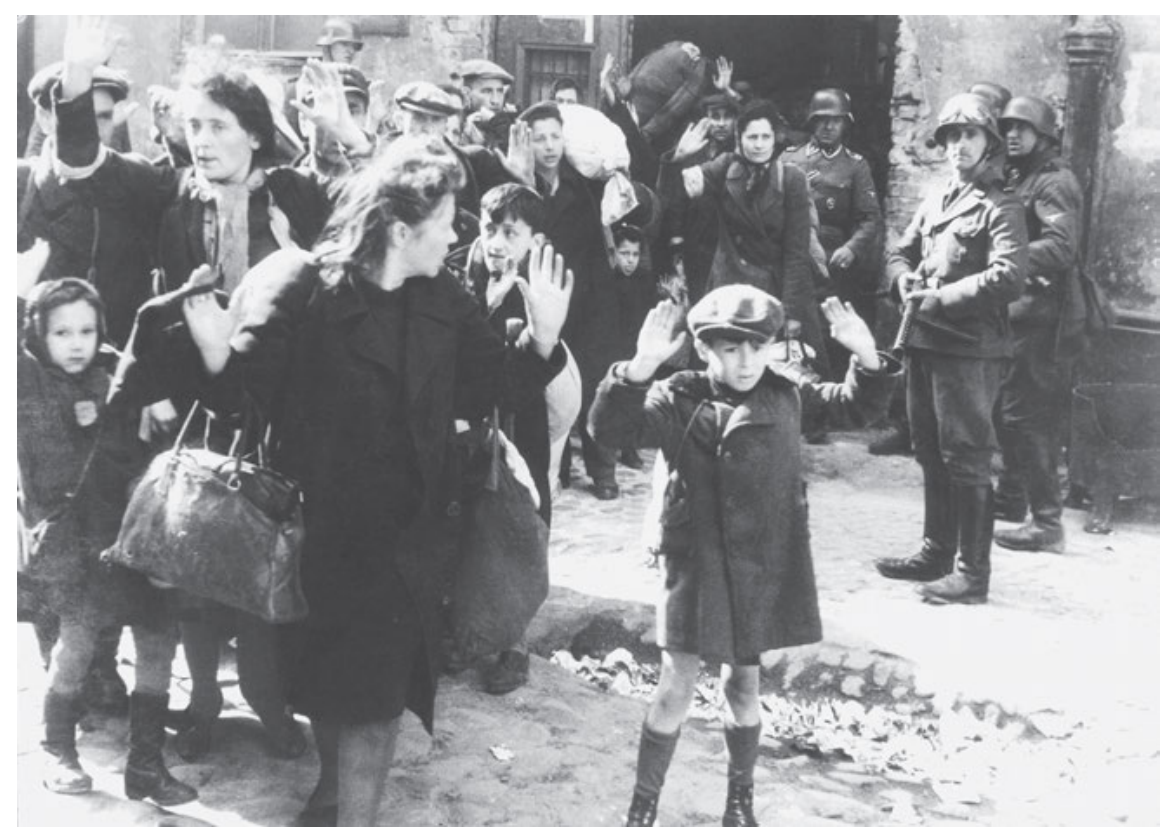

Abb. 1: Fotografie aus dem sogenannten Stroop-Bericht

czapce z daszkiem i w podkolanówkach, z podniesionymi do góry rękami“ (Rymkiewicz 1992, 244) [„,Ein Bild, das alle kennen: ein Junge mit Schirmmütze und Kniestrümpfen, die Arme erhoben“ (Rymkiewicz 1993, 325)]. Anders als in zahlreichen künstlerischen Rekursen auf diese Fotografie ${ }^{8}$ ändert sich das Umfeld des Abgelichteten hier nicht (Abb. 1), ${ }^{9}$ sondern es wird im Gegenteil ausführ-

8 Der inflationäre Gebrauch dieses Kamerazeugnisses, das dem Berichtsalbum Es gibt keinen jüdischen Wohnbezirk in Warschau mehr des SS-Generals Jürgen Stroop entstammt (Brink 1998, 107-112), wurde etwa von dem französischen Historiker Frédéric Rousseau ebenso eindrücklich wie umfangreich dokumentiert (2012). Vgl. etwa auch den Umschlag zu Ghetto Warschau: Aufstand und Vernichtung 1943 - fünfzig Jahre danach zum Gedenken (Wiehn 1993). Zur künstlerischen Bearbeitung vgl. exemplarisch Judy Chicagos Im/Balance of Power von 1991 (http://www. judychicago.com/gallery/holocaust-project/hp-artwork/, 20. September 2015) oder Samuel Baks Crossed Out II von 2007 (https://www.kunst-archive.net/en/wvz/samuel_bak/works/icon_of_ loss_crossed_out_ii/type/all, 20. September 2015).

9 Im Gegensatz zu einigen anderen Abgelichteten ist die Identität des Jungen (vgl. http://www. deathcamps.org/occupation/gunpoint_de.html) ebenso ungeklärt wie die des Fotografen (vgl. Struk 2004, 82-83, 200-201; Raskin 2004). Im Bericht lautet die Bildunterschrift „Mit Gewalt aus Bunkern hervorgeholt“ (https://de.wikipedia.org/wiki/Datei:Stroop_Report_-_Warsaw_Ghetto_ Uprising_BW.jpg sowie https://archive.org/details/STROOPBericht/page/n202). 
lich geschildert (Rymkiewicz 1992, 244-245; Rymkiewicz 1993, 326-327). Zudem erlangt der Junge in Umschlagplatz eine namentliche Biografie ${ }^{10}$ als ebenso wie der Ich-Erzähler 1935 geborener Artur.

Artur jest moim rówieśnikiem [...]. Stoimy obok siebie, on na tym zdjęciu zrobionym w warszawskim getcie, a ja na zdjęciu zrobionym na wysokim peronie w Otwocku. [...] On w podkolanówkach, ja w białych skarpetkach. (Rymkiewicz 1992, 245)

Artur ist mein Altersgenosse [...]. Wir stehen nebeneinander, er auf diesem Bild, das im Warschauer Ghetto gemacht wurde, und ich auf dem Bild, das auf dem hohen Perron in Otwock aufgenommen wurde. [...] Er trägt Kniestrümpfe, ich weiße Socken. (Rymkiewicz 1993, 327-328)

Das zuvor fotografisch Absente verdichtet sich in der Shoah-Ikone des Gleichaltrigen, dem der Ich-Erzähler mit seiner Aufnahme vom Ferienbahnhof in sekundärer und hierbei einzig lichtbildlich motivierter Augenzeugenschaft gegenübertritt. Nachträglich weist sich der Ich-Erzähler in der fiktiven Fotocollage jedoch als Augenzeuge aus, der nicht wegsieht, sondern emphatisch agiert:

- Zmęczyłeś się - mówię do Artura. - To przecież musi być bardzo niewygodne: takie stanie z podniesionymi do góry rękami. To zróbmy tak. Teraz ja podniosę ręce, a ty je opuścisz. I może oni tego nie zauważą. Albo wiesz, co. Zrobimy inaczej. Obaj będziemy stać z podniesionymi do góry rękami. (Rymkiewicz 1992, 246)

Du bist müde, sage ich zu Artur. Das muß doch sehr anstrengend sein: die ganze Zeit mit erhobenen Armen dazustehen. Machen wir das so. Nun werde ich die Arme heben, und du nimmst sie herunter. Vielleicht bemerken sie das gar nicht. Oder, weißt du was. Machen wir es anders. Wir stehen beide mir erhobenen Armen da. (Rymkiewicz 1993, 328)

Die imaginierte Anrede des Getto-Jungen suggeriert, dass sein verschonter Altersgenosse ihm die Last der Unterdrückung und Bedrohung zwar nicht abnehmen, sie jedoch mit ihm teilen kann. Dass dieser etwas schwülstige Wiedergutmachungsversuch mittels einer fiktiven Fotocollage erfolgt, weist die so unterschiedlichen Ablichtungen als genauso differente apparative Augenzeugnisse aus. Sie können - je nach Betrachtung - ebenso eine Evidenz des Gräuels, jedoch auch seiner Abwesenheit aufweisen wie eine Latenz, in der gerade das durchschimmert, was im fotografischen Off liegt. Narrativ gewinnen sowohl der Abzug aus dem persönlichen Fotoalbum als auch das ikonische Shoah-Bild ein derartiges

10 Nach heutigen Erkenntnissen ist die Identität des Jungen nicht eindeutig feststellbar. Die von Rymkiewicz zugeschriebene Biografie von Artur Siemiątek ist Raskin (2004) zufolge eine von vier Möglichkeiten. 
Eigenleben, dass sie sich gegen Textende quasi-filmisch überblenden. Indem dies imaginativ und damit für das retinale Sehen unsichtbar geschieht, wird bei der Überblendung mit der bekannten Getto-Aufnahme gerade die fotografische Latenz profiliert. Im Entwicklerbad der Dunkelkammer ist hiermit eben die spurenhafte, noch verschwommene Sichtbarwerdung des Abgelichteten gemeint.

Im Gegensatz hierzu steht die Bilderflut zum Gräuel der Shoah, deren Entdeckung für Susan Sontag in On Photographie (1977) noch ein Schlüsselerlebnis darstellt, wenn sie als Zwölfjährige KZ-Aufnahmen aus Bergen-Belsen und Dachau entdeckt: „Nichts, was ich jemals gesehen habe, - ob auf Fotos oder in der Realität - hat mich so jäh, so tief und unmittelbar getroffen“ (1995, 25-26). Retrospektiv ergänzt Sontag allerdings: „Hat man einmal solche Bilder betrachtet, dann ist man bereits auf dem Weg, mehr davon zu sehen - und immer mehr. Bilder lähmen. Bilder betäuben“ $(1995,26)$. Eben dieser nachlassenden Schockwirkung werden in Umschlagplatz die Bildränder entgegengesetzt, die im Familienalbum dem widersprechen, was fotografisch ansonsten in Überfülle dokumentiert wurde. Die Abzüge schockieren den Selbst-Betrachter in Umschlagplatz deswegen, weil das Medium der apparativen Augenzeugenschaft die Gräueltaten ausspart, was eine Besprechung, teils geradezu Beschwörung latenter Opferbilder aus dem Off initiiert und in narrativen Konjunktiven kompensiert.

\section{Opfer-Erbschaften durch latent erahnte Zeugungsakte}

In Sie kam aus Mariupol von 2017 spekuliert Natascha Wodin, Jahrgang 1945, ${ }^{11}$ über das Schicksal ihrer ukrainischen Mutter, die sich, als die Autorin elf Jahre alt war, das Leben nahm:

Sie hatte vor über neunzig Jahren gelebt, nicht irgendwelche Jahre, sondern die Jahre des Bürgerkriegs, der Säuberungen und Hungerkatastrophen in der Sowjetunion, die Jahre des Zweiten Weltkriegs und des Nationalsozialismus. Sie war in den Reißwolf zweier Diktaturen geraten, zuerst unter Stalin in der Ukraine, dann unter Hitler in Deutschland. (Wodin 2017, 10)

11 Die deutsche Schriftstellerin und Literatur-Übersetzerin aus dem Russischen ist Tochter von Zwangsarbeitern aus der Ukraine und heißt eigentlich Natalja Vdovina. Nach Kriegsende lebte sie mit ihrer Familie unter verheerenden Verhältnissen in deutschen Lagern. Für Sie kam aus Mariupol wurde sie 2017 mit dem Preis der Leipziger Buchmesse ausgezeichnet. 
Der Leitspruch ihrer als Opfer unterschiedlicher Regime traumatisierten Mutter lautet „Wenn du gesehen hättest, was ich gesehen habe...“ (Wodin 2017, 319). Eben dieser im Konjunktiv gehaltenen Aussparung widmet sich der Großteil des Textes, denn die Tochter stößt bei einer zunächst eher spielerischen Internetrecherche nach der Mutter tatsächlich auf einen Eintrag:

Als ich diesen Namen in einer Sommernacht des Jahres 2013 ins russische Internet eingegeben hatte, lieferte mir die Suchmaschine prompt ein Resultat. [...] Ich öffnete den Link und las: Iwaschtschenko, Jewgenia Jakowlewna, Geburtsjahr 1920, Geburtsort Mariupol. Ich starrte auf den Eintrag, er starrte zurück. (Wodin 2017, 10-11)

Dieser digitale Fund löst über Hunderte von Buchseiten eine Ansammlung neuer Fakten, Dokumente, Schriften und Fotos sowie digitale Begegnungen mit der russisch-ukrainischen Verwandtschaft aus, was den Stammbaum einer aristokratischen Familie erstellen lässt (Wodin 2017, 367-368). Durch eben dieses multimediale Archiv schließen sich zahlreiche Lücken derjenigen Erinnerungen, die vor der elterlichen Ankunft in Deutschland liegen. Über die folgende Zeit bis zum Tod der Mutter 1956 erfährt Wodin am wenigsten: „In meiner Erinnerung war sie nur noch ein Schemen, mehr ein Gefühl als eine Erinnerung“ (2017, 10). Hierzu gehören einschneidende Erlebnisse wie Schläge des alkoholisierten Vaters (2017, 307-308), Gewaltausbrüche, bei denen die verzweifelte Mutter ihre Tochter physisch angreift (2017, 351), sexueller Missbrauch durch einen Lagerinsassen (2017, 318-319) oder das schmerzhafte Außenseitertum in der deutschen Volksschule (2017, 332-336). „In den Tiefen [...] [ihrer] Erinnerung“ (2017, 306) verortet Wodin Übergriffe, die sie als Kind nicht versteht und durch die sie den elterlichen Opfer-Status ,erbt'. Durch diese erschreckende Übertragung verletzen die durch Zwangsarbeit in Deutschland und den späteren Aufenthalt im Valka-Lager schwer beschädigten Eltern ihre eigene Tochter physisch wie psychisch. Als aufschlussreich erweist sich dabei, dass die Autorin explizit darüber spekuliert, wie sich dieses Erbe bereits in den eigenen Zeugungsakt einschrieb:

Ich stelle mir vor, es ist ein Sonntag, der Tag, den die meisten Arbeiter zum Schlafen nutzen. [...] Aber dieser Sonntag im frühen März, der Frühling liegt schon in der Luft, ist ein Festtag für meine Eltern. Sie haben Ausgang bekommen und verlassen gemeinsam das Lagergelände. [...] Beide tragen das vorgeschriebene Abzeichen ,OST ' [...]. Vielleicht passiert es an diesem Tag, vielleicht finden sie irgendwo in den Ruinen ein Versteck. Vielleicht bin ich aber auch das Resultat einer gehetzten, atemlosen Umarmung irgendwo im Lager, wo sie jeden Augenblick entdeckt werden können, womöglich gewittert von einem der Schäferhunde, mit deren Hilfe das Wachpersonal nach Flüchtlingen sucht. Vielleicht ist meine Zeugung einem Moment des Leichtsinns geschuldet, weil bereits das Ende des Krieges in der Luft liegt. (Wodin 2017, 281-283) 


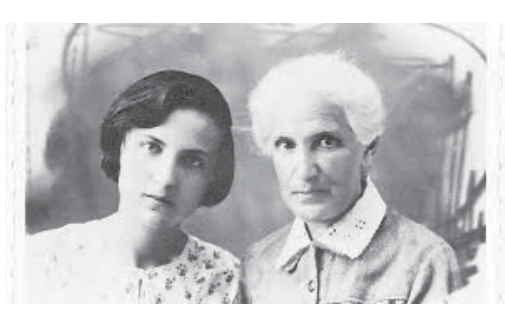

Abb. 2: Wodins weißhaarige Großmutter und dunkelhaarige Mutter, ca. 1938

Die wiederholt als Möglichkeiten - „Ich stelle mir vor“, „vielleicht“ - geschilderten Zeugungsumstände stehen dem Faktenwissen, dessen digitales Zusammentragen von einem Mecklenburger See aus ausgiebig geschildert wird, als konjunktivische Einblendung entgegen (Hufen 2017). Analog zur ,Selbstzeugung ${ }^{*}$ löst der überraschend auftauchende Lebensbericht von Wodins Tante Lidia Spekulationen über die Zeugung der Mutter aus, die in einer Bürgerkriegsnacht 1919 während eines Besuchs des Großvaters bei seiner Frau vermutet wird:

Ein fünfundfünfzigjähriger Mann und eine zweiundvierzigjährige Frau, deren Haar über Nacht weiß geworden ist vor Grauen, zeugen in einem Augenblick unvorsichtiger Selbstvergessenheit ein Kind. [...] Vermutlich sind sie ausgehungert nach einander, vielleicht denken sie, dass sie sich zum letzten Mal in den Armen halten. (Wodin 2017, 181)

Bedeutsam ist hierbei, dass die Autorin zuvor durch die Aufzeichnungen ihrer Tante von einem Einbruch ins großelterliche Haus erfährt, bei dem die Weißgardisten eine Waffe auf die Großmutter richteten (Wodin 2017, 180). Die hierbei erlebte Todesangst lässt sie über Nacht ergrauen (Wodin 2017, 181), wodurch sich für die Enkelin nunmehr das Rätsel über

die weißhaarige Frau auf dem Foto mit meiner jungen Mutter [löst]. Matilda war schon weißhaarig, als sie meine Mutter mit dreiundvierzig Jahren zur Welt brachte. Eine weißhaarige Gebärende, eine Weißhaarige, die einen Säugling stillt. Davor hatte sie wahrscheinlich schwarzes Haar gehabt, wie meine Mutter. (Wodin 2017, 181)

Das entsprechende Foto (Abb. 2) zeugt nicht nur von einer grausamen Vorgeschichte, die sich um das Ergrauen der Großmutter dreht, sondern weist hierdurch eine Latenz auf, die Schilderungen eines möglichen Zeugungsakts, der gleichfalls unter den Vorzeichen erlittener Gewalt steht, auslöst. Im Gegensatz hierzu steht das Internet, das als Medium der sekundären Oralität Verbindungen zur entfernten mütterlichen Verwandtschaft herstellt, der die Autorin auf digitalem Wege begegnet und deren Dokumente die „Blackbox“ (Wodin 2017, 53) der frühen Familiengeschichte öffnen. Dagegen erweisen sich die deutsche Zwangsarbeiterepisode und das Leiden als Fremde kurz nach Kriegsende sowie in der 
,jungen' Bundesrepublik als ähnlich spekulativ wie die erwogenen Umstände der mütterlichen und eigenen Zeugung vor der Folie von Opfernarrativen, die eine entsprechende Erbschaft ankündigen. Dass fiktionale Selbstdarstellungen mitunter abstruse Züge annehmen, zeigt Wodins amüsanter Hinweis auf die ,russische“ Selbsterfindung Iwan Rebroffs, mit dem ihr Vater in einem Kosakenchor sang. In der Bundesrepublik galt Rebroff „als Verkörperung der russischen Seele [...], obwohl er mit Russland rein gar nichts zu tun hatte. Er war ein Deutscher, der weder Iwan noch Rebroff hieß, sondern Hans Rippert“ (Wodin 2017, 349). Wodin wird als Schulkind nicht nur gehänselt, sondern sogar gedemütigt:

[D]ie Lehrerin, eine germanische Blondine mit stahlblauen Augen, die nie den Rohrstock aus der Hand legt und nicht mit den gefürchteten Tatzen spart, ist kein Schutz für mich, im Gegenteil. Mit ihren Erzählungen von den Gräueltaten der Russen, von ihrer Mordgier und Brutalität, fordert sie meine Mitschüler geradezu dazu auf, über mich herzufallen. (Wodin 2017, 332)

Angesichts dieses Schulalltags verleugnet das Mädchen zwecks Selbstaufwertung ihre leiblichen Eltern und erfindet eine aristokratische Familiengeschichte: „[I]n Wirklichkeit würde ich aus einer reichen Fürstenfamilie stammen, die Schlösser und Güter besaß“ (Wodin 2017, 25). Jahrzehnte später bewahrheitet sich diese Selbsterfindung zum Teil. Demgegenüber bleibt die Zeit seit Ankunft der Eltern in Deutschland weitgehend im Dunkeln bzw. äußert sich in bruchstückhaften Traumatisierungen. Wie Böttiger (2017) in seiner Rezension feststellt, handelt es sich um ein historisches „Kapitel, das fast völlig unbekannt ist. Das angenähte Zeichen ,O` für ,Ostarbeiter` stand wie der gelbe Stern der Juden für schlimmste Unterdrückung und elendste Bedingungen“.

\section{Leere Fotoecken und historische Irritationen: Latenz-Effekte im Comic}

In ihrem Debüt-Comic Liebe schaut weg von 2007 wendet Line Hoven, Jahrgang 1977, ${ }^{12}$ eine aufwändige Schabtechnik an, indem sie Konturen in Papierbögen ritzt, die zuvor zunächst mit weißer Kreide, dann mit schwarzer Tusche beschichtet wurden. Diesem Verfahren kommt bei der Visualisierung von Erin-

12 Die deutsche Comic-Autorin studierte unter anderem an der Hamburger Hochschule für Angewandte Wissenschaft bei Anke Feuchtenberger und ATAK (Georg Barber). Für Liebe schaut weg (Hoven 2007), ihre Diplomarbeit, erhielt sie 2008 den Independent-Comic-Preis (Mahrt 2008). 
nerung besondere Bedeutung $\mathrm{zu}$, da das Abkratzen schwarzer Schabkartons gängigen Metaphern zum Vorgang des Erinnerns und Vergessens ähnelt, wie diese bereits in Platons Theätet als Wachstafel oder bei Freud als ebenso überschreibbarer wie löschbarer Wunderblock mit Spuren (des Unbewussten) vorgestellt werden (Felka 2004). Vergegenwärtigung von Vergangenem erfolgt in dem Comic in Weißschwarzkontrasten, durch weiße Striche, die penible Konturen erzeugen (Steinaecker 2017; Platthaus 2009; Kupczyńska 2013). Sichtbar werden dadurch Momentaufnahmen aus dem Familienarchiv der Autorin. Sie zeigen den Großvater väterlicherseits als Hitlerjungen, den Großvater mütterlicherseits als kriegswilligen amerikanischen Patrioten, das Kennenlernen der Eltern und deren Familien in Bonn sowie die junge Familie bis in die 1970er Jahre vor jeweils zeittypischen Kulissen. Visuell entsteht durch die Schabtechnik ein fotogleicher und damit latent wirkender Negativ-Effekt, der den Comic mit einem Fotoalbum analogisiert und hiervon zugleich absetzt. Schwarz sind auch die meist geometrischen Sprechblasen, die oft fast unmerklich in die Panels integriert sind und gerade dadurch irritieren.

Durch die fotografischen Anleihen referiert der Comic autoreflexiv auf seinen medienhistorischen Status als Nachfolgemedium, das sich aber gerade im Gegensatz zur - wenn letztlich auch vermeintlichen - fotografischen Realitätsabbildung durch zeichnerisches Handwerk samt (Hand)Schrift vom apparativ erzeugten Lichtbild unterscheidet und dadurch das Fiktive der Narrationen geradezu ausstellt. ${ }^{13}$ Eben diese mediale bzw. remediale, also auf das Vorgängermedium referierende Folie nutzt Hoven für ihre postmemoriale Erzählung in Liebe schaut weg, wodurch das Dargestellte stets im Konjunktiv bleibt. ${ }^{14}$

Erkennbar ist dies vor allem an Schriftstücken und Fotos, die einerseits als dokumentarische Reliquien der Familiengeschichte fungieren, andererseits Zeichnungen sind, für die es möglicherweise keine Vorlagen gibt. Auffallend in den Blick geraten diese als Quasi-Titel der jeweiligen Kapitel, die deren Inhalte

13 Platthaus konstatiert grundlegend: „Es ist kein Zufall, dass es Comics erst seit etwas mehr als hundert Jahren gibt, denn zuvor entstand kein Bedarf an dieser Erzählform. Dann aber, als Bilder zu identischen Darstellungen des Wirklichen werden konnten, bekam die graphische Erzählform ihren Reiz“ (2009, 6). Der Medientheoretiker Marshall McLuhan insistiert noch allgemeiner darauf, dass zum Verständnis eines Mediums das Wissen um seine „relations to other media, old and new“ $(1994,202)$ unerlässlich ist. Auch seine Nachfolger in der New Media Theory betonen „the representation of one medium in another“ (Bolter und Grusin 1999, 45), wobei das jeweils neuere Medium gerechtfertigt sei, „because it fills a lack or repairs a fault in its predecessor“ (Bolter und Grusin 1999, 60).

$14 \mathrm{Zu}$ einem ähnlichen konjunktivischen Erzählverfahren bei Marcel Beyer und Paweł Huelle vgl. Heidemann 2017. 


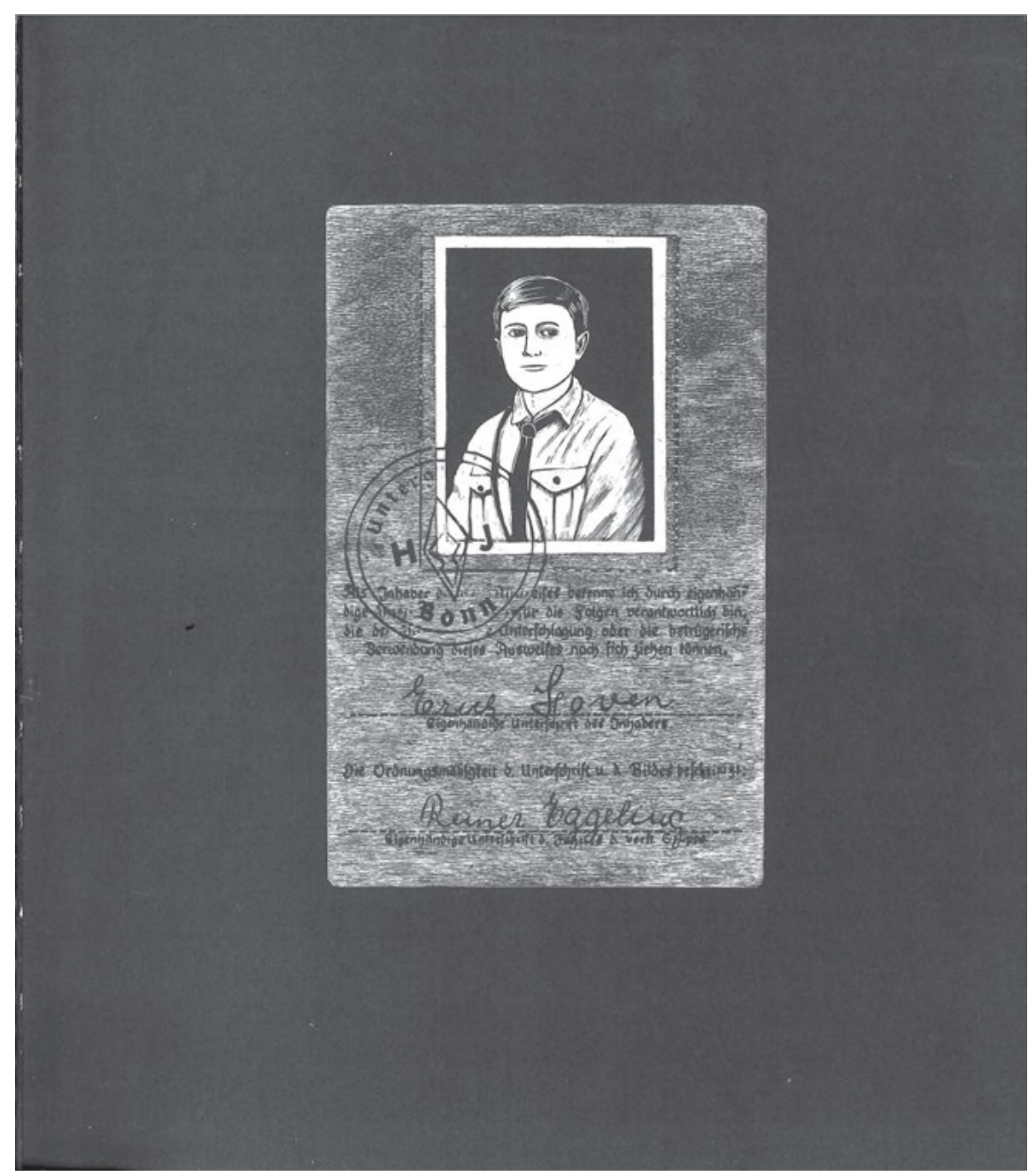

Abb. 3: Hitlerjugend-Ausweis von Erich Hoven

thematisch komprimieren. Dem ersten Kapitel wird entsprechend der großväterliche Hitlerjugend-Ausweis (Abb. 3) vorangestellt, dessen gemaserte Detailansicht im Stempel sogleich den Handlungsort Bonn erkennen lässt. Bezeichnenderweise endet dieses Kapitel mit einer Doppelseite, die einen Blick ins Familienalbum gewährt, in dem ausgerechnet das Foto fehlt, das „Erich \& Irmgard im Sommerlager der Hitlerjugend“ zeigt. Dort lernte sich, wie die Bildunterschrift suggeriert, das spätere Ehepaar kennen, das eine mehrköpfige Familie gründete, in der Richard 1945 als erstes Kind geboren wurde. Die gekennzeichneten Fotos 


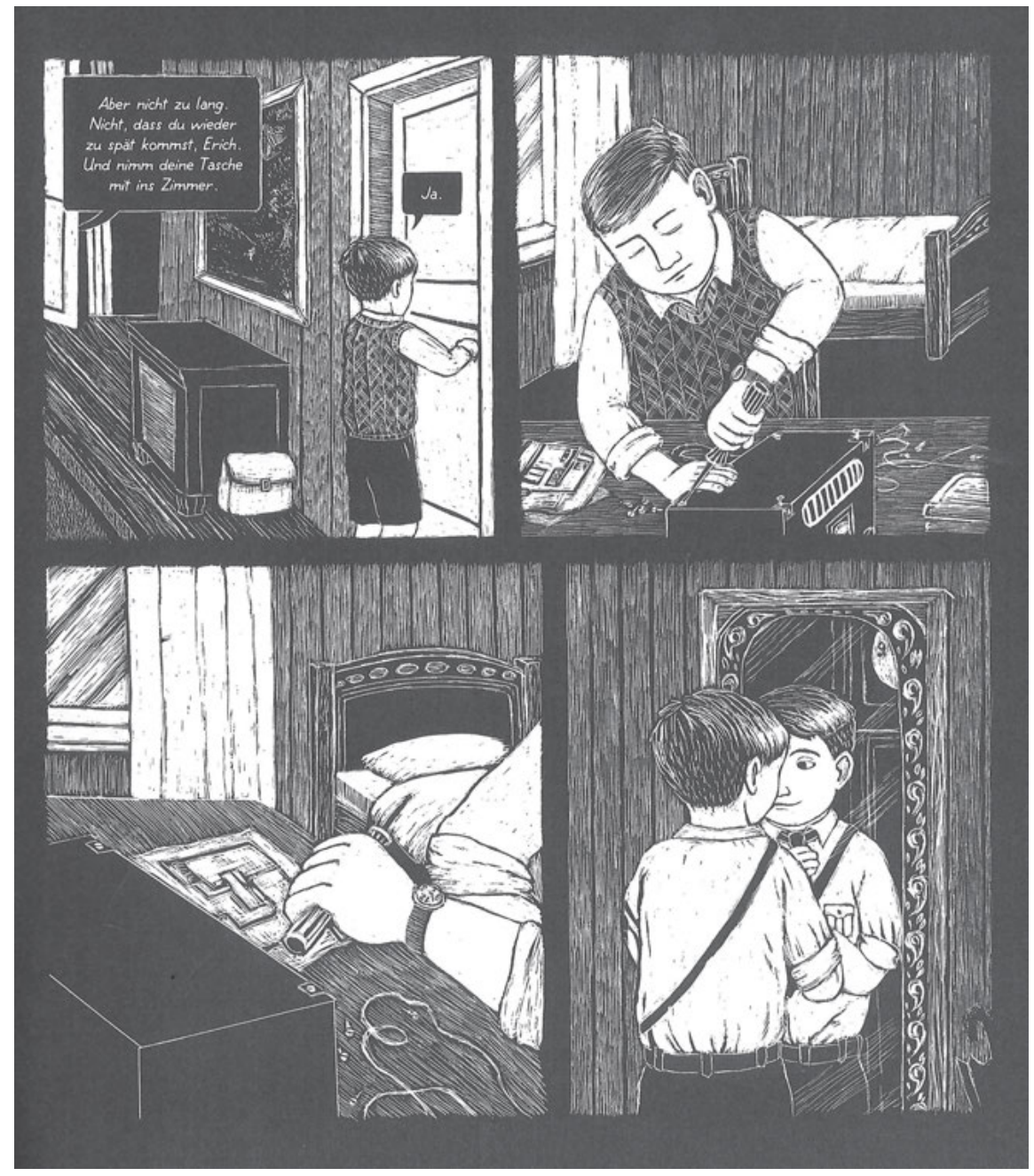

Abb. 4: Erichs Radiopassion

markieren allerdings nicht nur familiäre Schlüsselmomente, sondern stellen diese bzw. das fotografische Familienarchiv als solches ebenso in Frage, indem verwaiste Fotoecken samt Bildunterschrift auf zufällige, absichtliche oder fragliche Ausblendungen verweisen. Zudem kompensieren die Fotos Zeitsprünge in der fragmentarischen Narration, deren Ausblendungen zugleich Lücken im Familiengedächtnis darstellen können.

Retrospektiv verweisen gerade die verwaisten Fotoecken auf eine latent bleibende Erinnerung, die den Großvater der Autorin väterlicherseits betrifft. Im 


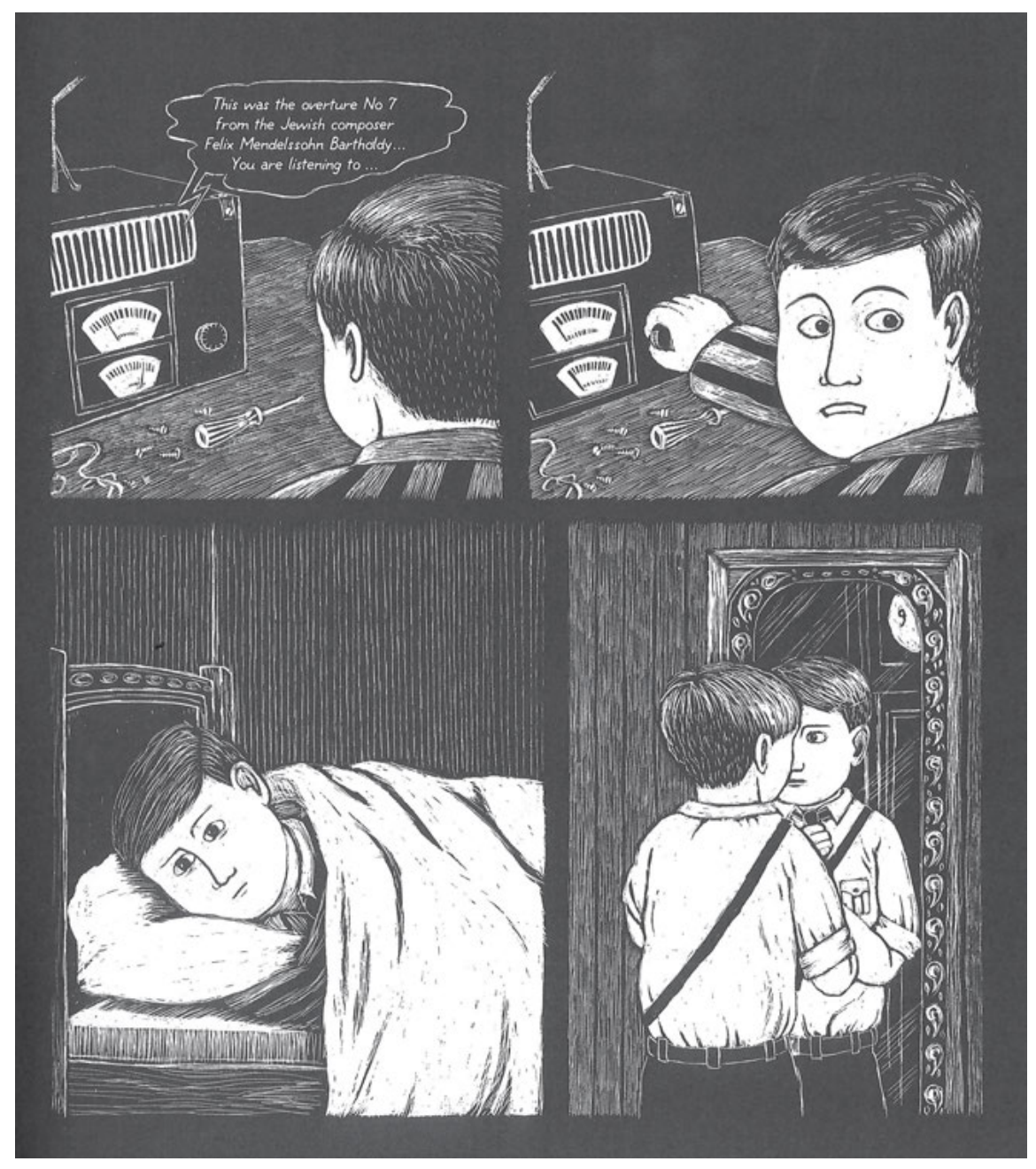

Abb. 5: Folgenschweres Lauschen einer verbotenen Mendelssohn-Ouvertüre

Comic zeigt ein wiederholt in Kopfnähe des jungen Erich Hoven auftauchender Schaltplan seine Technikbegeisterung an, die ihn so beschäftigt, dass er, wie suggeriert wird, fast seine Pflichten in der Hitlerjugend vergisst (Abb. 4).

Abends bastelt der Junge an einem Radiogerät, bis es tatsächlich funktioniert. Folglich schwelgt Erich in der Musikübertragung, über die im Radio dann folgendermaßen informiert wird: „This was the overture No 7 from the Jewish composer Felix Mendelssohn Bartholdy.“ Wie an den folgenden Panels ablesbar ist, erschreckt den Jungen das Gehörte dermaßen, dass er nicht einschlafen 
kann (Abb. 5). Ob dies an dem verbotenen Hören des Feindsenders liegt oder daran, dass die zuvor noch genossene Musik von einem jüdischen Komponisten stammt, bleibt unklar. Offensichtlich wird im vierten Panel der Seite allerdings, dass die auditive Grenzüberschreitung Erich an seine Pflichten als Hitlerjunge erinnert, weshalb er vor dem Spiegel seine Uniform richtet - eine Art Selbstkontrolle. Während der Junge sich selbst hier versteinert ins Auge blickt, lächelt er in einem vorherigen Parallelpanel seinem uniformierten Spiegelbild zu (Abb. 4). Unentschieden bleibt im Vergleich, worauf die physiognomische Veränderung zurückzuführen ist - auf den Schock, dass wegen der ideologischen Verbote nunmehr der Experimentiererfolg und seine Früchte hinfällig sind, oder auf einen Quasi-Lauschangriff aus dem Ausland. Wenn Erich später von gleichaltrigen Kameraden gefragt wird, wie es um sein Radio steht, verkündet er kurz und bündig: „Das ist kaputt.“ Bemerkenswert ist hierbei der Hintergrund, der leere Schaufensterauslagen mit Aufschriften wie „Jud verrecke“ unter einem Davidstern zeigt, worauf Erich ängstlich zu blicken scheint (Abb. 6). Damit liegt in dieser Episode eine aufschlussreiche Latenz vor, die hier die Unzuverlässigkeit der Familienerinnerung markiert. So weist Kalina Kupczyńska darauf hin, dass „Mendelssohn in England vor dem Krieg als protestantischer Komponist bekannt und gefeiert [wurde]“ $(2013,196)$. Die betreffende Episode bezieht sich allerdings auf die 1930er Jahre. ${ }^{15}$ Das für den im Nationalsozialismus aufgewachsenen Erich Hoven einschneidende Erlebnis widerspricht hier nicht den historischen Tatsachen, um die großväterliche Erinnerung als falsche aufzudecken oder sogar anzuklagen, sondern um grundsätzlich zeitliche Verschiebungen, Fehler und Unzuverlässigkeiten beim Erinnern einzuräumen. ${ }^{16}$ Auf einer Metaebene wird damit auch reflektiert, dass gerade in der postmemorialen Erinnerung an das großelterliche Leben im Nationalsozialismus Täter- und Opferschaft nicht immer eindeutig zu trennen sind. So kann Erichs Radio-Episode ebenso auf eine Schutzbehauptung zurückgehen, womit der Großvater seinen ,kleinen' Widerstand als Junge rühmt und die Indoktrinierung der Jugend anklagt. Ebenso gut kann sich aber hinter dem ,jüdischen Lauschangriff‘ eine ideologische Zustimmung verbergen, wie sie in der Hitlerjugend gelernt wurde. Entsprechend deuten auf möglicherweise unbeliebte Themen in der Familiengeschichte explizite Auslassungen

15 Auch Andreas Platthaus merkt an: „Erst später, im Krieg, stellte die BBC auf Propaganda um und betonte bewusst in den Sendungen für Deutschland die jüdische Abstammung großer Künstler“ (2009, 6).

16 Ähnlich unterstreicht Kupczyńska: „Das Phänomen des Vergessens reflektiert man hier als einen genuinen Bestandteil der Erinnerungsprozesse und als einen Faktor der Konstituierung des Gedächtnisses“ $(2013,197)$. 


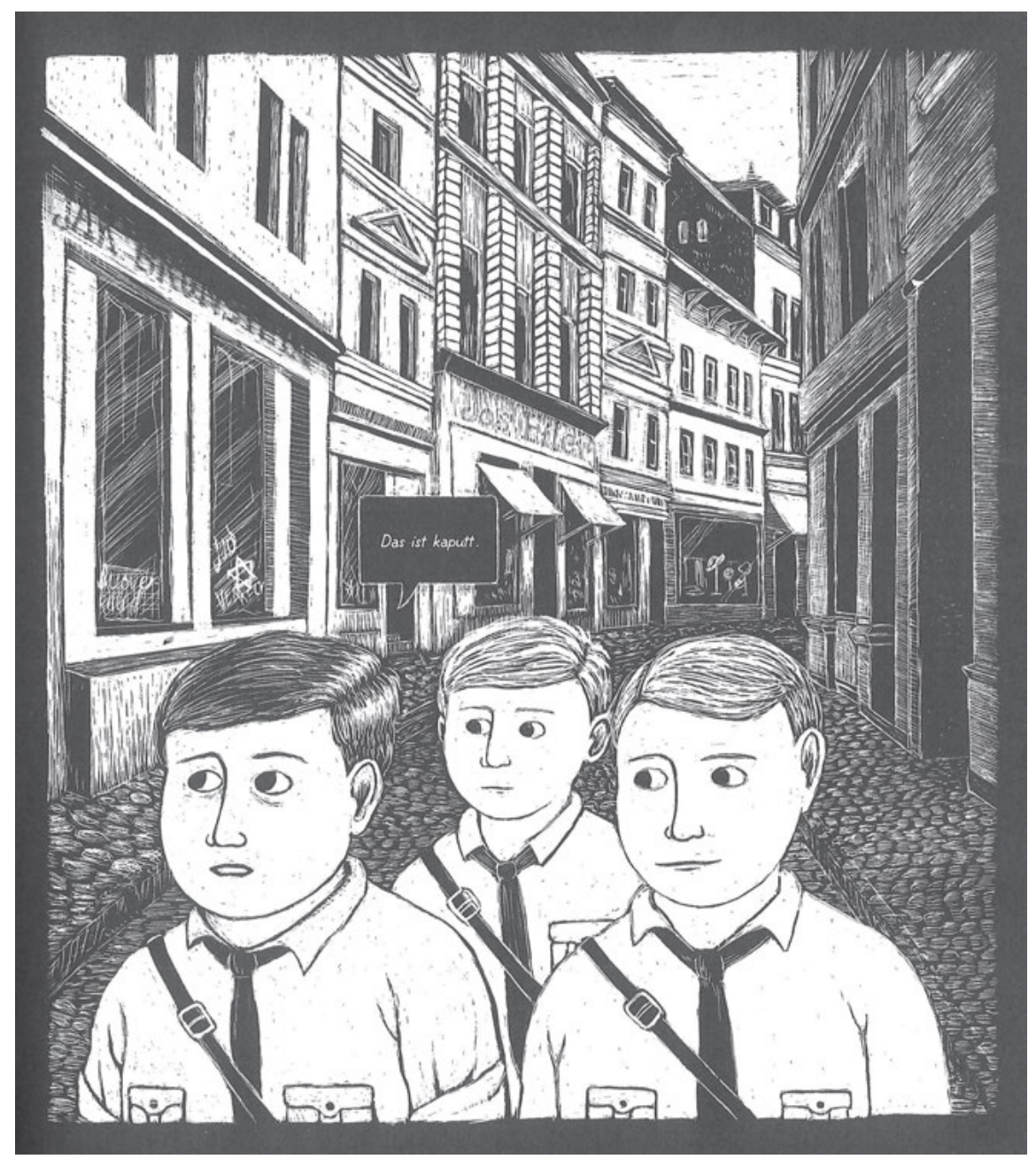

Abb. 6: Kaputtes Radio, leere Schaufenster

hin. So antwortet Richard, der erwachsene Sohn Erichs, wenn er angesichts eines Fotos seines Vaters in Wehrmachtsuniform nach dem Kennenlernen seiner Eltern gefragt wird: „Ich glaube, es war in der Hitlerjugend. Sommerlager oder so... Sie reden nicht über diese Zeit. Ist schon lange her." Wird das Lückenhafte zunächst durch die verwaisten Fotoecken illustriert, kommt es hier eher verbal zum Ausdruck, was ebenso auf das grafisch-verbale Zusammenspiel im Comic verweist wie auf seine elliptische und eingefrorene Erzählweise.

Vor allem Letztgenanntes verdichtet sich zu Beginn des Comics prologartig in einem verlassenen Zimmer (Abb. 7) mit Umzugskartons, leeren Wänden, an 


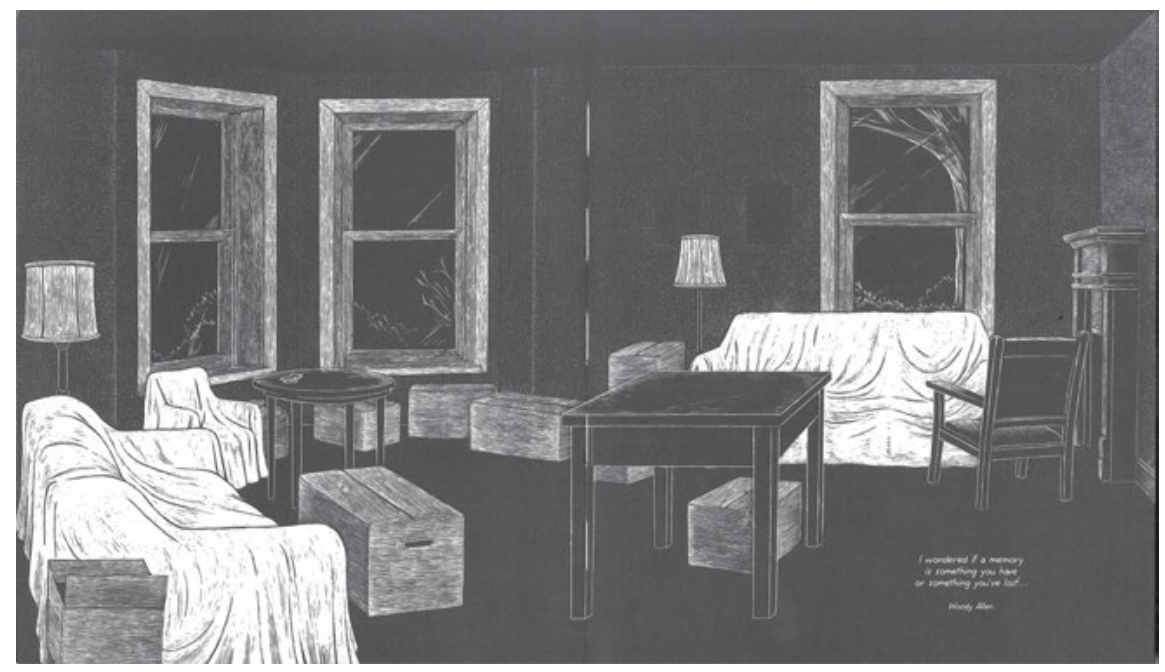

Abb. 7: Verlassenes Zimmer als Prologbild

denen erkennbar ist, wo zuvor Bilder hingen, weißen Laken über den Polstermöbeln und einem Zitat in der rechten unteren Ecke: „I wondered if a memory is something you have or something you've lost ...“, das aus Woody Allens Film Another Woman stammt. Angesichts der Radioepisode läge es aber näher, dass es sich um ein Zitat aus Radio Days handelt, worin die Kindheitserinnerungen eines Off-Erzählers farbenfroh ins Bild gesetzt werden und dabei Anekdoten rund um die Radioproduktion und -rezeption in New York Ende der 1930er, Anfang der 1940er Jahre erzählt werden. In den Rückblicken gerät das damals sich verbreitende Medium zum Leitmotiv von Erinnerungen, bei denen vor allem ,auditive، Kanäle angesprochen werden. Auch diese falsche Fährte dient einer Irritation, die Erinnerungen abermals als unzuverlässig ausweist. Hiermit und mit dem Prologbild selbst signalisiert der Comic bildlich wie verbal, dass die Familienerinnerung durch Spuren von Vergessenem, durch Abwesendes, Latentes und Präsentes bedingt ist. Ausgelassene Momente der Vergangenheit werden als solche gezeigt oder angedeutet, was ein bruchstückhaftes Erzählen bewirkt. Indem das menschenleere Zimmer auch unheimlich wirkt, zitiert es die Verschränkung des Heimischen, Heimlichen und Unheimlichen in Sigmund Freuds psychoanalytisch-etymologischer Interpretation ${ }^{17}$ und unterstreicht damit räumliche Erinnerungsaspekte. Diese illustriert kurz darauf der großväterliche Haus-

17 Diese erfolgte 1919 mit Bezug auf E. T. A. Hoffmanns Sandmann-Erzählung (Freud 2000). 


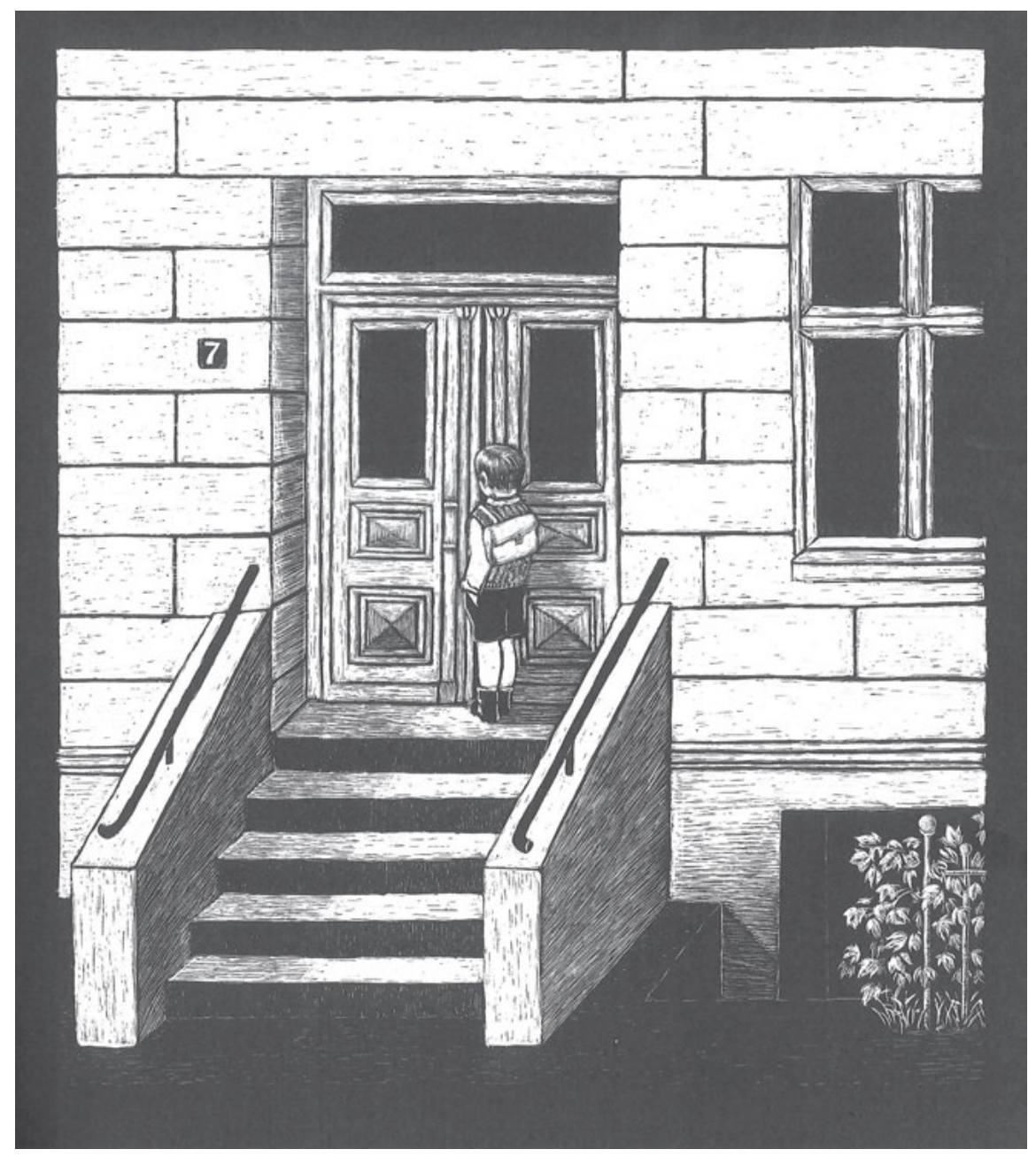

Abb. 8: Hauseingang der großväterlichen Kindheit

eingang in Bonn (Abb. 8), der am Ende nach der Rückkehr von Richards junger Familie aus den USA nochmals samt Erichs amerikanischer Schwiegertochter und seiner Enkelin ins Blickfeld gerückt wird (Abb. 9). Hierin liegt auch einer der Gründe, warum das Prologbild als einziges nicht gerahmt ist, d.h., dass es in zeitlicher Entgrenzung ebenso der Kindheit des Großvaters - ob als verschreckter oder begeisterter Hitlerjunge - wie der Kindheit der Enkelin zugeordnet werden 


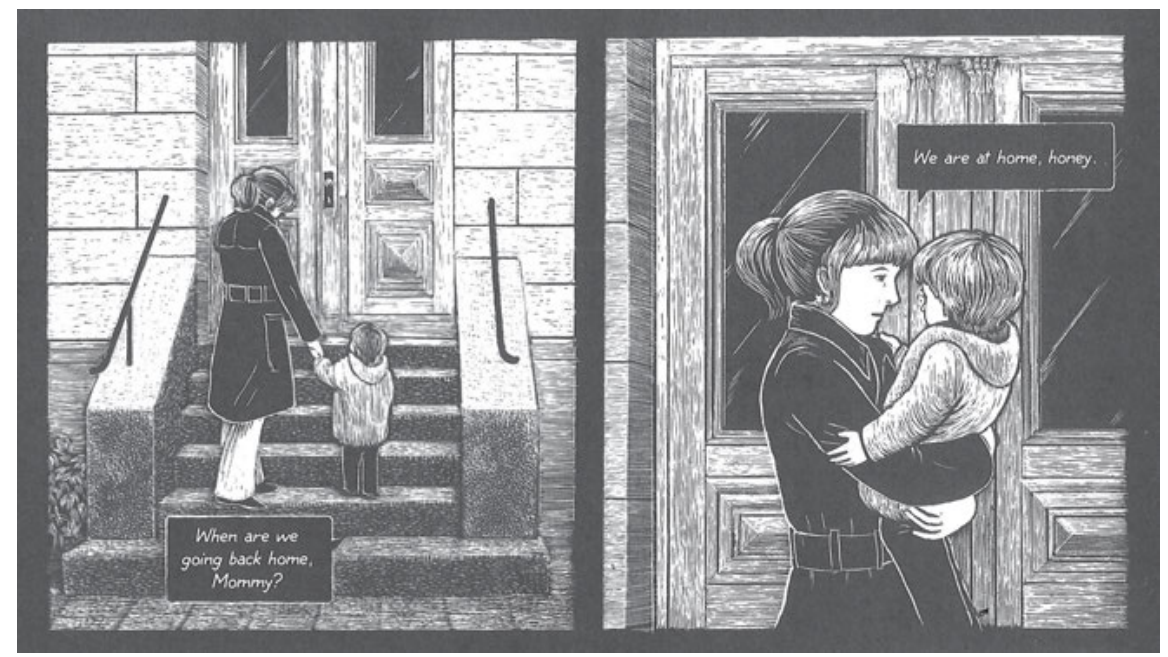

Abb. 9: Hauseingang der Enkelin-Kindheit

kann. ${ }^{18}$ Erinnerung wird mit dem Hauseingang und dem verdunkelten Zimmer vor allem räumlich verortet, ${ }^{19}$ was der bildlichen Anlage des Comics entspricht. Wenn Liebe schaut weg jeweils zeitgenössisch populäre Medien (Zierold 2006, 183) zitiert, bleibt gemäß dem illustrativen Erfindungsmodus die tatsächliche familiäre Teilhabe an den Medienereignissen offen. So mag auch die RadioRezeption ebenso zum Bild gewordene Vorstellung der Autorin wie mediengesteuerte familiäre bzw. großväterliche Einbildung sein, was hier letztlich auch die Trennung zwischen Opfer- und Täterschaft verschwimmen lässt.

Das als Gemeinplatz daherkommende,Wegschauen' im etwas kitschig anmutenden Comictitel Liebe schaut weg erweist sich damit als programmatisch, denn dies betrifft nicht nur die Familienepisoden im Nationalsozialismus, in den USA und in der ,jungen' Bonner Bundesrepublik, sondern auch die explizit visualisierten Auslassungen wie impliziten Erfindungen. Durch die Illustrationen des, Wegschauens', die Lücken im Familienalbum, die Bildspuren im verlassenen Zimmer werden ein Vergessen sowie eine Latenz ins Bild gesetzt, deren Gründe unklar bleiben. Deutlich zeigen vor allem die Familienfotos als geschabte ,Quasi-Nega-

18 So betont Scott McCloud: „Die nicht länger von dem gewohnten Symbol, dem geschlossenen Panelrahmen, eingegrenzte Zeit entrinnt in den zeitlosen Raum“ (2001, 107).

19 Kupczyńska ergänzt diesbezüglich aufgrund des Filmzitats: „Und - das filmische Medium transportiert unterschwellig die ihm inhärente Verquickung von Zeit und Raum, die für jede Erinnerungsarbeit konstitutiv ist“ $(2013,194)$. 


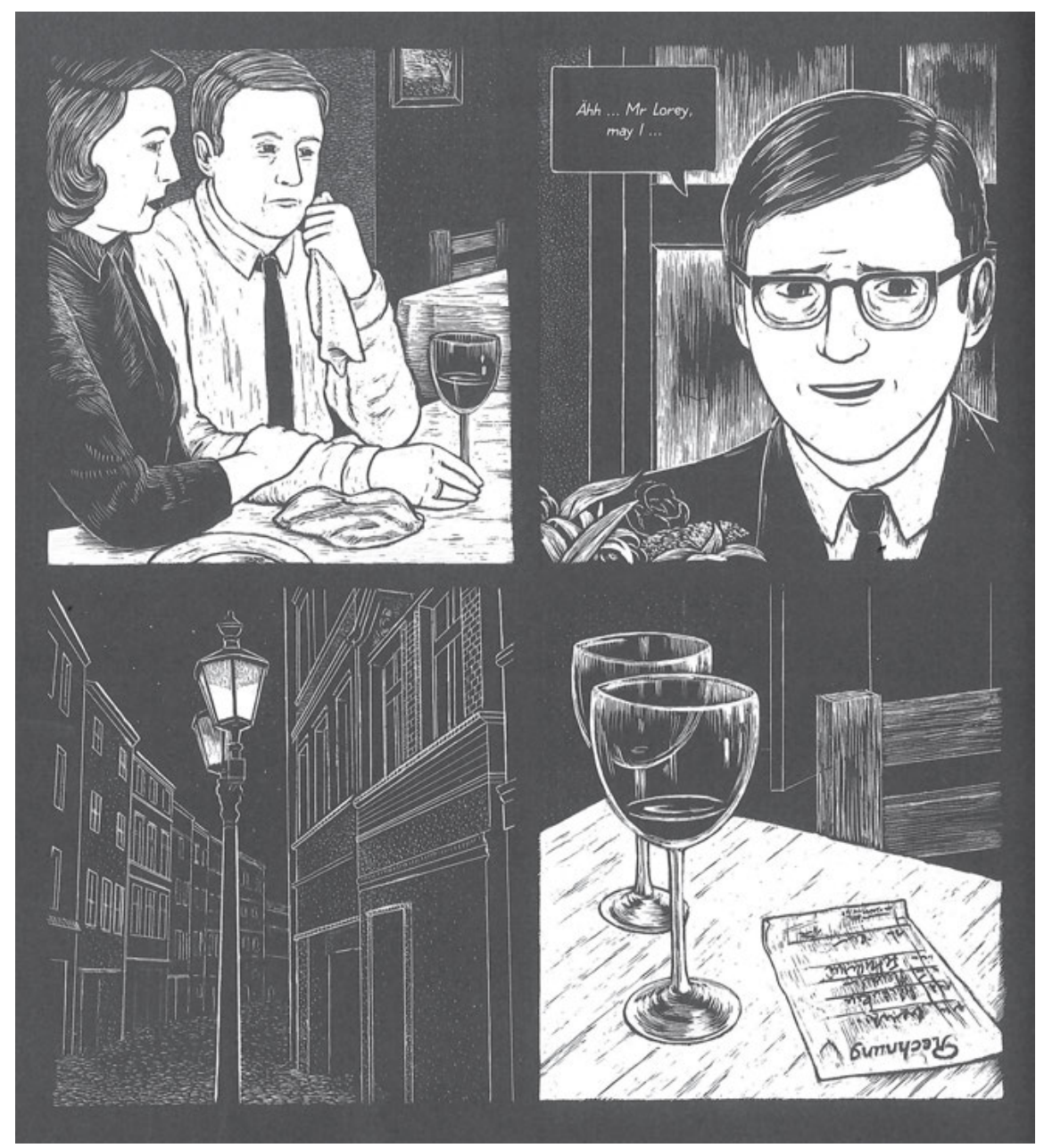

Abb. 10: Lückenhaftes Handanhalten

tive' ein Erinnern auf, das eben nicht analog zur Schwarzweißmalerei erfolgt, sondern in Weißschwarzkontrasten die Schichten des Erinnerns und Vergessens aufschabt. In Hovens postmemorialer Erinnerung bleibt sogar offen, ob die Auslassungen im Familiengedächtnis auf die (Groß-)Eltern zurückgehen oder von der Autorin selbst vorgenommen wurden. Daher kann sich hinter dem marschierenden Hitlerjungen und dem jungen Wehrmachtssoldaten ebenso ein Opfer verbergen wie umgekehrt hinter dem auf technische Neuerungen seiner Zeit fixierten Jungen ein williger Pflichterfüller. Von einer solchen Unzuverlässigkeit zeugt vor allem das Latente, das hier etwa aufgrund zeitverschobener Zuordnungen his- 
torischer Umstände auszumachen ist, sodass die Gründe für den Schrecken des Jungen unklar bleiben, oder auf die Irritation aufgrund eines fehlenden Filmzitats zur Kopplung von Erinnerungen an die Anfänge des Radios zurückgehen.

An den Bildrändern von Kindheitsfotografien aus glücklichen Sommertagen macht Rymkiewicz Altersgenossen aus, die zeitgleich in nahe gelegenen Gettos eingesperrt waren und damit latent im Familienalbum aufblitzen. Narrativ werden die jungen Opfer auf Fotos entdeckt und aufbewahrt, deren Ferienposen im Wissen um die Dokumentation „des Dagewesenseins“ (Barthes 1990, 39; Bourdieu 1981, 31) ${ }^{20}$ den erhobenen Händen auf mehr oder minder bekannten Gettofotografien vehement widersprechen. Auch bei Wodin ist es die Latenz der Fotografie, die die junge Mutter ${ }^{21}$ neben einer ergrauten Frau zeigt, die ein weiteres Opfer in der eigenen Genealogie darstellt. Vor allem aber initiiert die Autorin die bewusste Erfindung intimer Zeugungsakte als eine Opfer-Erbfolge, die bis zu ihr selbst reicht. Hoven dagegen kalkuliert eine Unzuverlässigkeit in der Familienerinnerung ein, die ebenso Opfer-Täter-Zuschreibungen betrifft. Daher erweist sich auch die Episode, in der der Medizinstudent Richard um die Hand der amerikanischen Studentin Charlotte, Tochter des einst kriegswilligen amerikanischen Patrioten, anhält, als lückenhaft (Abb. 10). Im ersten Panel schaut Charlottes Vater jemanden unzufrieden an, ihre Mutter beobachtet angestrengt, im zweiten Panel stammelt Richard „Ähh ... Mr Lorey, may I ...“, im dritten Panel erscheint eine dunkle menschenleere Gasse und im vierten Panel sind zwei Weingläser, eines mit einer ,traurigen Neige‘, sowie eine Rechnung und ein leerer Stuhl zu sehen. Die Szenerie wirkt gerade durch die ausgesparten Momente des Handanhaltens und der offensichtlichen, aber eben nicht gezeigten Ablehnung bedrückend. Von diesen Auslassungen zeugen neben dem Schweigen und Stottern in den letzten beiden Panels die Visualisierung des Verlassenen und insgesamt die abrupten Bildwechsel. Letztgenannte erinnern zugleich an filmische Bildsprünge, wodurch ebenso ein gezieltes Wegschauen in den unangenehmsten Augenblicken erfolgt wie eine fotoanaloge Erstarrung als visuelle Fixierung, was das Geschehen dramatisiert, indem es derart entschleunigt zugleich Schock-

20 Konrad Köstlin zufolge „sind wir zu Historikern unserer selbst geworden“ (1995, 399).

21 Die auch fotografische Suche nach der Mutter erinnert an Barthes' Die helle Kammer (1985), worin der Autor in seiner Trauer um den Tod der Mutter eine authentische Ablichtung zu finden hofft, die nicht nur die gewesene Existenz bestätigt, sondern die Mutter so zeigt, wie er sich an sie erinnert. Von einer solchen ,Lichtspur‘ zeugt schließlich ein nicht abgedrucktes Wintergartenfoto, das Barthes' Mutter als Fünfjährige zeigt (Barthes 1985, 77-78) und das in der zweiten Essayhälfte im Fokus seiner fotografischen Re-Visionen steht. Zentral ist auch in W. G. Sebalds Austerlitz (2001, 350-352) der Versuch, die Mutter mittels filmischer Standbilder quasi-fotografisch ausfindig zu machen. 
momente aus der Perspektive des jungen Paares erwägt. Wir haben es hierbei mit einer Übertragung von direkt oder indirekt Kriegsinvolvierten auf die Generation der Kinder zu tun, wobei sich nunmehr das deutsch-amerikanische Paar als Opfer erweist. Die medialen Transfers, die im Comic durch Strategien der Remedialisierung - etwa von apparativ erzeugten Fotos in handwerklich hergestellte Comics - vorgenommen werden, bilden das Transgenerationale auf einer Metaebene ab, die selbst auf das für den Comic konstitutive Zusammenspiel von Wort, Schrift und Illustration verweist.

\section{Literaturverzeichnis}

Assmann, Aleida. „Zur Mediengeschichte des kulturellen Gedächtnisses“. Medien des kollektiven Gedächtnisses: Konstruktivität - Historizität - Kulturspezifität. Hg. Astrid Erll und Ansgar Nünning. Berlin und New York: De Gruyter, 2004. 45-60.

Assmann, Aleida. Der lange Schatten der Vergangenheit: Erinnerungskultur und Geschichtspolitik. Bonn: bpb, 2007.

Assmann, Aleida, und Ute Frevert. Geschichtsvergessenheit - Geschichtsversessenheit: Vom Umgang mit deutschen Vergangenheiten nach 1945. Stuttgart: Deutsche Verlagsanstalt, 1999.

Barthes, Roland. „Historical Discourse“. Structuralism: A Reader. Hg. Michael Lane. London: Jonathan Cape, 1970. 154-155.

Barthes, Roland. Die helle Kammer: Bemerkung zur Photographie. Übers. von Dietrich Leube. Frankfurt a. M.: Suhrkamp, 1985.

Barthes, Roland. Der entgegenkommende und der stumpfe Sinn: Kritische Essays III. Übers. von Dieter Hornig. Frankfurt a. M.: Suhrkamp, 1990.

Böttiger, Helmut. „Natascha Wodin: Dann spielt die Mutter Chopin“. DIE ZEIT, 9. März 2017, https://www.zeit.de/2017/11/natascha-wodin-sie-kam-aus-mariupol (10. Oktober 2017).

Bolter, Jay David, und Richard Grusin. Remediation: Understanding New Media. Cambridge, Mass.: MIT Press, 1999.

Bourdieu, Pierre. Eine illegitime Kunst: Die sozialen Gebrauchsweisen der Photographie. Übers. von Udo Rennert. Frankfurt a. M.: Europäische Verlagsanstalt, 1981.

Brink, Cornelia. Ikonen der Vernichtung: Öffentlicher Gebrauch von Fotografien aus nationalsozialistischen Konzentrationslagern nach 1945. Berlin: Akademie-Verlag, 1998.

Chaumont, Jean-Michel. Die Konkurrenz der Opfer: Genozid, Identität und Anerkennung. Übers. von Thomas Laugstien. Lüneburg: zu Klampen, 2001.

Didi-Huberman, Georges. Bilder trotz allem. Übers. von Peter Geimer. München: Fink, 2007.

Diekmann, Stefanie, und Thomas Khurana. „Latenz: Eine Einleitung“. Latenz: 40 Annäherungen an einen Begriff. Hg. Stefanie Diekmann und Thomas Khurana. Berlin: Kadmos, 2007. 9-13.

Felka, Rike. Psychische Schrift: Freud - Derrida - Celan. Weimar: Univ.-Verl., 2004.

Finkelstein, Norman G. The Holocaust Industry: Reflections on the Exploitation of Jewish Suffering. London und New York: Verso, 2000.

Finkelstein, Norman G. Die Holocaust-Industrie: Wie das Leiden der Juden ausgebeutet wird. Übers. von Helmut Reuter. München und Zürich: Piper, 2001. 
Franzen, K. Erik, und Martin Schulze Wessel. Hg. Opfernarrative: Konkurrenzen und Deutungskämpfe in Deutschland und im östlichen Europa nach dem Zweiten Weltkrieg. München: Oldenbourg, 2012.

Freud, Sigmund. „Das Unheimliche“. Sigmund Freud: Studienausgabe. Band 4. Hg. Alexander Mitscherlich, Angela Richards und James Strachney. Frankfurt a. M.: Suhrkamp, 2000. 241-274.

Gerstner, Jan. Das andere Gedächtnis: Fotografie in der Literatur des 20. Jahrhunderts. Bielefeld: transcript, 2013.

Giglioli, Daniele. Die Opferfalle: Wie die Vergangenheit die Zukunft fesselt. Übers. von Max Henninger. Berlin: Matthes \& Seitz, 2015.

Gisbertz, Anna-Katharina, und Michael Ostheimer. Hg. Geschichte - Latenz - Zukunft: Zur narrativen Modellierung von Zeit in der Gegenwartsliteratur. Hannover: Wehrhahn, 2017.

Heidemann, Gudrun. „Between Ethos and Pathos: Missing and Lasting Exposures in Marcel Beyer's Spies (Spione) and Paweł Huelle's Mercedes-Benz: From Letters to Hrabal (Mercedes-Benz: Z listów do Hrabala)“. Ethos und Pathos: Mediale Wirkungsästhetik im 20. Jahrhundert in Ost und West. Hg. Riccardo Nicolosi und Tanja Zimmermann. Wien u.a.: Böhlau, 2017. 289-306.

Heindl, Nina, und Véronique Sina. „Repräsentation ,trotz allem““. Notwendige Unzulänglichkeit: Künstlerische und mediale Repräsentationen des Holocaust. Hg. Nina Heindl und Véronique Sina. Berlin und Münster: LIT, 2017. 1-11.

Hirsch, Marianne. Family Frames: Photography, Narrative and Postmemory. Cambridge, Mass.: Harvard University Press, 1997.

Hirsch, Marianne. „Surviving Images: Holocaust Photographs and the Work of Postmemory“. Visual Culture and the Holocaust. Hg. Barbie Zelizer. New Brunswick: Rutgers University Press, 2001. 215-246.

Hirsch, Marianne. The Generation of Postmemory: Writing and Visual Culture after the Holocaust. New York: Columbia University Press, 2012.

Hoven, Line. Liebe schaut weg. Berlin: Reprodukt, 2007.

Hufen, Uli. „Was kann ein Mensch ertragen?“ Deutschlandfunk, 26. Februar 2017, https://www. deutschlandfunk.de/roman-sie-kam-aus-mariupol-was-kann-ein-mensch-ertragen.700. de.html?dram:article_id=379905 (10. Oktober 2018).

Khurana, Thomas. „Latenzzeit: Unvordenkliche Nachwirkung: Anmerkungen zur Zeitlichkeit der Latenz“. Latenz: 40 Annäherungen an einen Begriff. Hg. Stefanie Diekmann und Thomas Khurana. Berlin: Kadmos, 2007. 142-147.

Köstlin, Konrad. „Photographierte Erinnerung? Bemerkungen zur Erinnerung im Zeitalter ihrer technischen Reproduzierbarkeit“. Hören, Sagen, Lesen, Lernen: Bausteine zu einer Geschichte der kommunikativen Kultur. Hg. Ursula Brunold-Bigler und Hermann Bausinger. Bern u.a.: Peter Lang, 1995. 395-410.

Kupczyńska, Kalina. „Ein-räumen, ver-innern: Topologie der Erinnerung in Line Hovens Graphic Novel Liebe schaut weg“. Topographie und Raum in der deutschen Sprache und Literatur. Hg. Fabrizio Cambi und Wolfgang Hackl. Wien: Praesens, 2013. 190-200.

Mahrt, Nina. „Liebe schaut weg von Line Hoven: Ein Interview“. Comic! Jahrbuch 2009. Hg. Burkhard Ihme. Stuttgart: Icom, 2008. 168-173.

McCloud, Scott. Comics richtig lesen: Die unsichtbare Kunst. Übers. von Heinrich Anders. Hamburg: Carlsen, 2001.

McLuhan, Marshall. Understanding Media: The Extensions of Man. Cambridge: MIT Press, 1994. Novick, Peter. The Holocaust in American Life. Boston: Houghton Mifflin Harcourt, 1999. 
Novick, Peter. Nach dem Holocaust: Der Umgang mit dem Massenmord. Übers. von Irmela Arnsperger und Boike Rehbein. Stuttgart und München: Deutsche Verlagsanstalt, 2001. Perechodnik, Calek. Czy ja jestem morderca? Warszawa: Karta, 1993.

Perechodnik, Calel. Bin ich ein Mörder? Das Testament eines jüdischen Ghetto-Polizisten. Übers. von Lavinia Oelkers. Springe: zu Klampen, 2015.

Platthaus, Andreas. „Anstelle einer Einleitung: Ein Motiv aus Line Hovens Liebe schaut weg“. Reddition 49/50 (2009): 5-7.

Raskin, Richard. A Child at Gunpoint: A Case Study in the Life of a Photo. Aarhus: Aarhus University Press, 2004.

Richter, Gerhard. „Man kann Auschwitz nicht abmalen“. FAZ, 25. Februar 2016, http://www. faz.net/aktuell/feuilleton/kunst/gerhard-richter-im-interview-ueber-gemaeldezyklusbirkenau-14088410.html?printPagedArticle=true\#void (13. September 2018).

Rousseau, Frédéric. Żydowskie dziecko z Warszawy: Historia pewnej fotografii [Ein jüdisches Kind aus Warschau: Geschichte einer Fotografie]. Übers. von Tomasz Swoboda. Gdańsk: stowo/obraz terytoria, 2012.

Rowiński, Tomasz. Spór o Rymkiewicza [Der Streit um Rymkiewicz]. Warszawa: Fronda, 2012. Rymkiewicz, Jarosław Marek. Umschlagplatz. Gdańsk: JMJ, 1992 [1988].

Rymkiewicz, Jarosław Marek. Umschlagplatz. Übers. von Martin Pollack. Berlin: Rowohlt, 1993. Sebald, W. G. Austerlitz. München: Hanser, 2001.

Sontag, Susan. Über Fotografie. Übers. von Gertrud Baruch und Mark W. Rien. Frankfurt a. M.: Suhrkamp, 1995.

Steinaecker, Thomas von. „In die Seele geritzt: Line Hoven debütiert mit einem FamilienComic“. Süddeutsche Zeitung, 20. November 2017 (Literaturbeilage).

Sterngast, Tal. „Bilder zur Unzeigbarkeit der Shoah: Zwischen Glanz und Rauch“. Taz, 10. September 2017, http://www.taz.de/!5442936/ (13. September 2018).

Struk, Janina. Photographing the Holocaust: Interpretations of the Evidence. London: I. B. Tauris, 2004.

Szpilman, Władysław. Śmierć miasta: Pamiętniki Wtadystawa Szpilmana 1939-1945. [Der Tod einer Stadt: Erinnerungen von Władystaw Szpilman]. Warszawa: Wiedza, 1946.

Szpilman, Władysław. Der Pianist: Mein wunderbares Überleben. Übers. von Karin Wolff. Berlin: Ullstein, 2004.

Werz, Michael. Hg. Antisemitismus und Gesellschaft: Zur Diskussion um Auschwitz, Kulturindustrie und Gewalt. Frankfurt a. M.: Verlag Neue Kritik, 2014.

Wiehn, Erhard Roy. Ghetto Warschau: Aufstand und Vernichtung 1943 - fünfzig Jahre danach zum Gedenken. Konstanz: Hartung-Gorre, 1993.

Wodin, Natascha. Sie kam aus Mariupol. Reinbek bei Hamburg: Rowohlt, 2017.

Zierold, Martin. Gesellschaftliche Erinnerung: Eine medienkulturwissenschaftliche Perspektive. Berlin und New York: De Gruyter, 2006. 


\section{Filmverzeichnis}

Another Woman. USA 1988. Regie: Woody Allen. Länge: 84 Min.

Finsterworld. Deutschland 2013. Regie: Frauke Finsterwalder. Länge: 85 Min.

Pianista [Der Pianist]. Frankreich, England, Deutschland und Polen 2002. Regie: Roman

Polanski. Länge: 150 Min.

Poeta pozwany [Angeklagter Poet]. Polen 2012. Regie: Grzegorz Braun. Länge: 56 Min.

Radio Days. USA 1982. Regie: Woody Allen. Länge: 84 Min.

\section{Abbildungsverzeichnis}

Abb. 1: Foto aus dem Stroop-Bericht. Wikimedia Commons, https://de.wikipedia.org/ wiki/Datei:Stroop_Report_-_Warsaw_Ghetto_Uprising_BW.jpg (10. Oktober 2018).

Abb. 2: $\quad$ Foto aus Wodin, Natascha. Sie kam aus Mariupol. Reinbek bei Hamburg: Rowohlt, 2017. 9.

Abb. 3-10: Panels und Panelsequenzen aus Hoven, Line. Liebe schaut weg. Berlin: Reprodukt, 2007. 
